\title{
On the packing measure of the Sierpinski gasket
}

\author{
Marta Llorente ${ }^{1}$, M. Eugenia Mera ${ }^{2}$ and Manuel Morán ${ }^{3}$. \\ ${ }^{1}$ Departamento de Análisis Económico: Economía Cuantitativa. \\ Universidad Autónoma de Madrid, Campus de Cantoblanco. \\ 28049 Madrid, Spain, E-mail: m.llorente@uam.es . \\ ${ }^{2,3}$ Departamento Fundamentos del Análisis Económico I. \\ Universidad Complutense de Madrid. Campus de Somosaguas. \\ 28223 Madrid, Spain, E-mails: ${ }^{2}$ mera@ucm.es, ${ }^{3}$ mmoranca@ucm.es
}

26 Februrary 2018

\begin{abstract}
We show that the $s$-dimensional packing measure $P^{s}(S)$ of the Sierpinski gasket $S$, where $s=\frac{\log 3}{\log 2}$ is the similarity dimension of $S$, satisfies $1.6677 \leq P^{s}(S) \leq 1.6713$.

The formula presented in Theorem 6 enables the achievement of the above measure bounds for this non-totally disconnected set as it shows that the symmetries of the Sierpinski gasket can be exploited to simplify the density characterization of $P^{s}$ obtained in [33] for self-similar sets satisfying the so-called Open Set Condition. Thanks to the reduction obtained in Theorem 6 we are able to handle the problem of computability of $P^{s}(S)$ with a suitable algorithm.
\end{abstract}

Keywords: Sierpinski gasket, packing measure, computability of fractal measures, algorithm, self-similar sets. Mathematics Subject Classification numbers: 28A75, 28A80.

\section{Introduction}

The aim of this paper is to determine upper and lower bounds for the packing measure $P^{s}(S)$ of the Sierpinski gasket. There are several results on bounds for the exact value of the Hausdorff measure of the Sierpinski gasket (see the main contributions in [32]), but as far as we know there are no such results for the exact value of the packing measure of the Sierpinski gasket.

In this introduction we give an overview of the main properties of the Sierpinski gasket and packing measures, and we discuss the issues involved in the computation of the packing measure of the Sierpinski gasket.

\subsection{The Sierpinski gasket. Hutchinson and Markov operators}

The Sierpinski gasket (see figure 1) or Sierpinski triangle, is one most popular mathematical and simplest geometrical objects having a fractal nature. Its particularly simple structure makes it a natural setting for testing and developing various mathematical and physical ideas, such as problems of fractal geometry, harmonic analysis, random walks and PDEs on fractals, networks, number theory, etc.; see [1],[3]-[6], chapter 12 of [7],[8], [11], [13], [19]-[23],[35],[39] and the references therein. Its pattern of construction seems to occur naturally to the human mind, at least in artistic contexts. Designs resembling a Sierpinski gasket appear in the 13th century in the floor mosaic of the central nave of the Roman Basilica of Santa Maria in Cosmedin and also in several churches, as an isolated Sierpinski gasket up to its third stage of construction. 


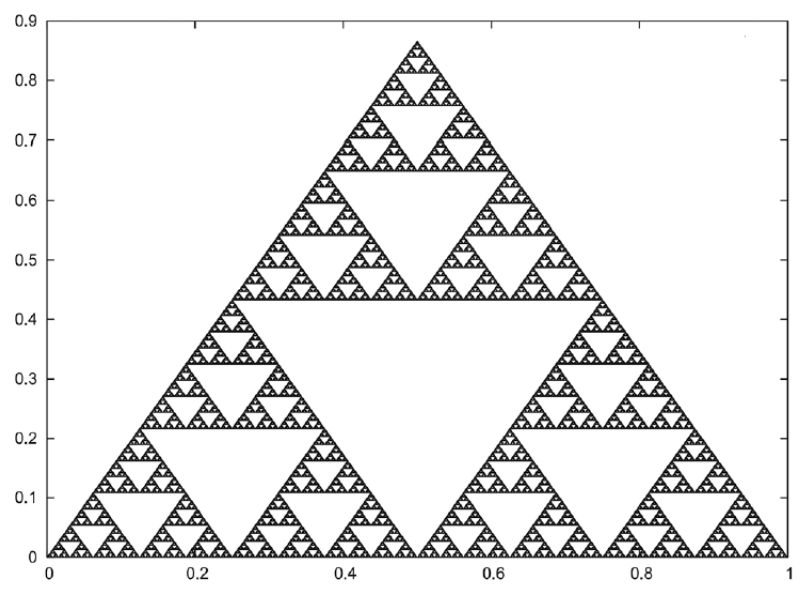

Figure 1. Sierpinski gasket.

The Sierpinski gasket was first described as a mathematical object by W. Sierpinski in 1915. It can be thought as a subset of the plane and as a mass distribution. As a subset of the plane its construction begins with a set $\Psi=\left\{f_{0}, f_{1}, f_{2}\right\}$ of three contracting similitudes of the plane, with contraction ratios $r_{i}:=1 / 2$, $i \in M:=\{0,1,2\}$, given by

$$
\begin{aligned}
f_{0}(x, y) & =\frac{1}{2}(x, y), \\
f_{1}(x, y) & =\frac{1}{2}(x, y)+\left(\frac{1}{2}, 0\right), \\
f_{2}(x, y) & =\frac{1}{2}(x, y)+\frac{1}{2}\left(\frac{1}{2}, \frac{\sqrt{3}}{2}\right) .
\end{aligned}
$$

The Hutchinson operator $F$, defined by

$$
F(A)=f_{0}(A) \cup f_{1}(A) \cup f_{2}(A), A \subset \mathbb{R}^{2},
$$

is useful in the analysis of the Sierpinski gasket as a set. It is a contracting operator on the space $\mathbf{H}\left(\mathbb{R}^{2}\right)$ of non-empty compact subsets of $\mathbb{R}^{2}$ endowed with the Hausdorff metric. The Sierpinski gasket $S$ is the unique fixed point of $F$, i.e., it is the unique non-empty compact set admitting the self-similar decomposition

$$
F(S)=S=f_{0}(S) \cup f_{1}(S) \cup f_{2}(S) .
$$

The set $S$ is known as the invariant set or attractor of $\Psi$.

That the Hutchinson operator is contracting means that, starting from any compact set, for instance, the set of three vertices $A_{1}=\left\{z_{0}, z_{1}, z_{2}\right\}$, there holds

$$
\lim _{k \rightarrow \infty} F^{k}\left(A_{1}\right)=S,
$$

where $F^{k}=F \circ F \circ \ldots \circ F$ is the $k$ th iterate of $F$ and the limit is with respect to the Hausdorff metric. Thus the Sierpinski gasket can be built with any desired level of detail by iterating the Hutchinson operator.

The Sierpinski gasket can be though also as a mass distribution $\mu_{S}$ supported on $S$, called the invariant or natural measure on $S$. The Markov operator $\mathbf{M}$, defined on the set $\mathcal{P}$ of Borel probability measures on $\mathbb{R}^{2}$ by

$$
\mathbf{M}(\nu)=\frac{1}{3} \sum_{i \in M} \nu \circ f_{i}^{-1}, \nu \in \mathcal{P}
$$

can be used to construct $\mu_{S}$. $\mathbf{M}$ is a contracting operator on $\mathcal{P}$ endowed with a suitable metric. Its $k$ th iterate $\mathbf{M}^{k}$ satisfies

$$
\mathbf{M}^{k}(\nu)=\left(\frac{1}{3}\right)^{k} \sum_{i \in M^{k}} \nu \circ f_{i}^{-1} \stackrel{w}{\rightarrow} \mu_{S},
$$




$$
\mathbf{M}\left(\mu_{S}\right)=\mu_{S}
$$

for any $\nu \in \mathcal{P}$, where $f_{i}:=f_{i_{1}} \circ f_{i_{2}} \circ \ldots \circ f_{i_{k}}$ for $i=i_{1} i_{2} \ldots i_{k} \in M^{k}$. Here $\stackrel{w}{\rightarrow}$ stands for the weak convergence. The natural measure $\mu_{S}$ is the unique fixed point of the Markov operator $\mathbf{M}$. Henceforth we shall write $\mu$ for the invariant measure $\mu_{S}$.

\subsection{The packing measure}

The packing and Hausdorff measures are the most popular metric measures used in describing the geometric properties of fractal sets. Given a subset $A \subset \mathbb{R}^{n}$, the $s$-dimensional Hausdorff measure detects the minimum $s$-volume of countable coverings of $A$. The dual concept of $s$-dimensional packing measure detects the maximum $s$-volume that can be covered by disjoint balls with centers in $A$.

Packing measures were introduced by Tricot [40]-[41], Taylor and Tricot [37]-[38], and Sullivan [36], as natural metric measures for analyzing Brownian paths and limit sets of Kleinian groups. Recall that a two-stage definition is needed for general Euclidean sets (see, for example,[29] or [30]). The situation for compact subsets is more manageable. The packing measure of a compact set $A \subset \mathbb{R}^{n}$ with finite packing premeasure is defined as

$$
P^{s}(A)=\lim _{\delta \rightarrow 0} P_{\delta}^{s}(A)
$$

where

$$
P_{\delta}^{s}(A)=\sup \left\{\sum_{i=1}^{\infty}\left|B_{i}\right|^{s}:\left|B_{i}\right| \leq \delta, i=1,2,3, \ldots\right\}
$$

is a non-decreasing set function with respect to $\delta$ and the supremum is taken over all countable $\delta$-packings based in $A$, i.e., all countable collections of disjoint Euclidean balls with centers in $A$ and with diameters smaller than $\delta$ (see [10]). From now on, given a subset $A \in \mathbb{R}^{n},|A|$ stands for its diameter. The $s$-packing measures of a set $A \subset \mathbb{R}^{n}$ have, like the Hausdorff measures, a threshold value $\operatorname{Dim}_{P}(A)$, called the packing dimension of $A$, such that $P^{s}(A)=0$ for $s>\operatorname{Dim}_{P}(A)$ and $P^{s}(A)=\infty$ for $s<\operatorname{Dim}_{P}(A)$.

In the case of self-similar sets, such as the Sierpinski gasket, the self-similarity can be used to express $P^{s}$ in terms of density functions. This facilitates addressing the computability problem algorithmically (see [24]-[28], [33] and [42]). Before such computational approaches were developed, the packing measure was known only for some particular cases of totally disconnected self-similar sets [2],[12], [17], [15].

In this paper we follow the computational approach in order to study the packing measure of the Sierpinski gasket. In particular, in [33], M. Morán obtained the following formula for the packing measure of self-similar sets that, as is the case for the Sierpinski gasket, satisfy the so-called open set condition, OSC in the sequel (see Section 1.3).

The open Euclidean ball centered at $x$ and with radius $d$ is denoted by $B(x, d)$.

Theorem 1 ([33]) Let $E$ be the invariant set of the system $\left\{f_{0}, \ldots, f_{N-1}\right\}$ of similarities satisfying the $\boldsymbol{O S C}$ for the set $\mathcal{O}$ with Hausdorff dimension $\operatorname{dim}_{\mathcal{H}} E=s$ and let $\mu$ be the normalized Hausdorff measure on $E$. Then

$$
P^{s}(E)=\sup \left\{\frac{(2 d)^{s}}{\mu(B(x, d))}: x \in E \text { and } B(x, d) \subset \mathcal{O}\right\} .
$$

Although, in general, this does not mean that the packing measure of $E$ can be computed easily, we shall see in Theorem 6 that, in the particular case of the Sierpinski gasket, (5) can be reduced to obtain a formula suitable for constructing an algorithm for approximating the value of its packing measure.

The idea behind this theorem is as follows. On the one hand, it can be proved that the inverse $s$-density, $h^{s}(B):=\frac{|B|^{s}}{\mu(B)}$, of any closed ball $B$ centered at $E$ provides a lower bound for $P^{s}(E)$. The proof is based on a construction of a suitable packing $\left\{B_{i}\right\}_{i \in \mathbb{N}}$ by balls centered at $E$ and obtained as images of $B$ under compositions of the similarities in $\left\{f_{0}, \ldots, f_{N-1}\right\}$. This construction can be done so the mentioned packing is also a $\mu$-almost all covering of $E$, i.e., $\sum_{i \in \mathbb{N}} \mu\left(B_{i}\right)=1$ and $h^{s}\left(B_{i}\right)=h^{s}(B)$ for every $i \in \mathbb{N}$. These properties lead to

$$
P^{s}(E) \geq \sum_{i \in \mathbb{N}}\left(2\left|B_{i}\right|\right)^{s}=h^{s}(B) \sum_{i \in \mathbb{N}} \mu\left(B_{i}\right)=h^{s}(B) .
$$

On the other hand, if there exists a ball $B^{*}$ maximizing (5), the standard method mentioned above can be used to construct an optimal packing from $B^{*}$. The exact value of $P^{s}(E)$ is then given by $h^{s}\left(B^{*}\right)$. 


\subsection{Computability of the packing measures of self-similar sets and the open set condition}

The existence of an optimal ball is a crucial requirement for the computability of $P^{s}(E)$. This requirement is fulfilled in the case of totally disconnected self-similar sets (see [33], [42], [24], [27], and [28]). For these fractals the minimum gap $c$ between the constituent parts, $f_{i}(E)$, of $E$ determines a lower bound for the radii of the balls $B(x, d)$ whose densities $\frac{(2 d)^{s}}{\mu(B(x, d))}$ are to be maximized.

The numerical method used for the computation of $P^{s}(E)$ is based on a discretization of the fractal, and the stage of construction required for that purpose depends on the smallest radii to be explored, so that if $c$ is small the computation of $P^{s}(E)$ requires so many iterations that it becomes too costly computationally.

The Sierpinski gasket is an example of a self-similar set that it is connected (so the above result does not apply in this case), but it satisfies the open set condition. This means that there exists an open set that will be, in our analysis, the rhombus $\mathcal{R}$ in figure $2(\mathrm{~b})$, satisfying $f_{i}(\mathcal{R}) \cap f_{j}(\mathcal{R})=\varnothing, i \neq j$, and $F(\mathcal{R}) \subset \mathcal{R}$. It is known [14], [30], that the packing dimension of $S, \operatorname{Dim}_{P}(S)$, coincides with the Hausdorff dimension and with the similarity dimension, $s=\frac{\log 3}{\log 2}$. Moreover, it can be shown in this case (see Remark 3 ) that both the packing and the Hausdorff measures are multiples of the natural measure $\mu$, i.e. there exist constants $C_{P}$ and $C_{\mathcal{H}}$ such that for any Borel set $A \subset S$,

$$
\begin{aligned}
& P^{s}(A)=C_{P} \mu(A), \\
& \mathcal{H}^{s}(A)=C_{\mathcal{H}} \mu(A),
\end{aligned}
$$

where $\mathcal{H}^{s}$ denotes the Hausdorff measure. The unknown constants $C_{P}$ and $C_{\mathcal{H}}$ are of course $P^{s}(S)$ and $\mathcal{H}^{s}(S)$, respectively. Knowledge of these constants makes the computation of the measures $P^{s}(A)$ and $\mathcal{H}^{s}(A)$ trivial for open subsets $A \subset S$ (see Remark 2). Therefore, since arbitrary subsets of $S$ can be approximated by open sets, the knowledge of $P^{s}(S)$ and $\mathcal{H}^{s}(S)$ solves the problems of computing the packing and Hausdorff measures on $S$.

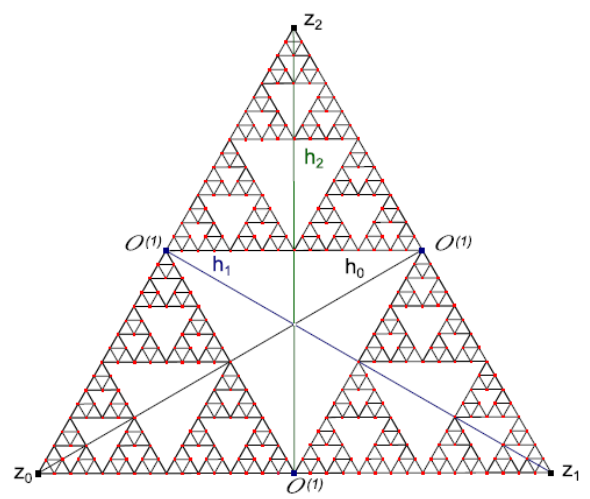

(a)

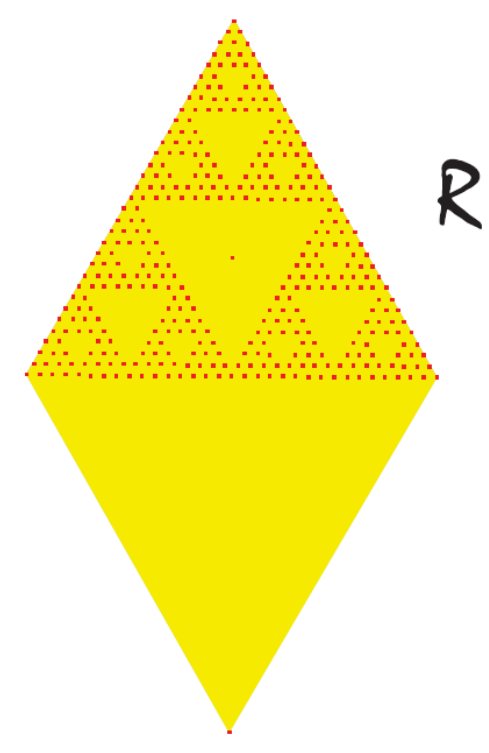

(b)

Figure 2. (a) Altitudes $h_{0}, h_{1}$ and $h_{2}$ of the triangle $T$ and the primary overlapping set $O^{(1)}$. (b) The rhombus $\mathcal{R}$ satisfying the OSC for the Sierpinski gasket.

Remark 2 Any open set $A \subset S$ can be written as a countable union of $\mu$-disjoint cylinders. From the knowledge of which cylinders in generation $k$ are contained in such a union, we can determine the value of $\mu(A)$ with arbitrary accuracy.

We show below that in the particular case of the Sierpinski gasket, the constant $C_{P}$ can actually be computed with some specified precision. The Sierpinski gasket does not fulfill the strong separation condition 
(totally disconnected case). The system of similarities $\Psi$ can be seen as the limit case, when $r$ tends to $1 / 2$, of systems $\Psi_{r}$ of three homotheties, having the same fixed points as $\Psi$, but with contraction ratios $r<1 / 2$. However, as $r$ tends to $1 / 2$ and the gap between the three copies under the similarities in $\Psi_{r}$ goes to zero, the computational time grows at a fatal rate, and the method used in the references mentioned above becomes useless (see Section 4.3 for further discussion). In fact linear self-similar sets and the $n$-dimensional unit cubes are the unique class of self-similar sets satisfying the OSC whose packing measure is known (see [9], $[10]$ and [31]).

Thus new ideas are required for the computation of $C_{P}=P^{s}(S)$. In this note it is shown that the existence of internal symmetries and homotheties in $S$ allows a reduction of the range of the radii of the balls to be explored that is similar to the reduction obtained in the totally disconnected case. As a matter of fact, with the results of this paper in hand, the packing measure of the attractors $S_{r}$ of the systems $\Psi_{r}$ with $r$ less than but close to $1 / 2$ is more difficult to compute than is $P^{s}(S)$ itself. The group generated by the symmetries with respect to the altitudes of the equilateral triangle $T$ with vertices in the fixed points of the similitudes in $\Psi$ and the homotheties with fixed points at the midpoints of the sides of $T$ and contraction ratio $1 / 2$ can be used to compute $P^{s}(S)$ approximately.

It should be remarked that the computation of the packing measure of a self-similar set $E$ satisfying the OSC is, in general, out of reach computationally. The computation of $C_{\mathcal{H}}=\mathcal{H}^{s}(S)$ and $\mathcal{H}^{s}(E)$ is in general a problem still harder than that of the computation of $P^{s}(S)$ and $P^{s}(E)$, respectively. See [44] and [45] for some of the known cases. In particular the computation of $\mathcal{H}^{s}(E)$ for a general connected self-similar set $E$ (with the exception of the unit $n$-dimensional cube) is also out of reach (see [16], [18] and [43] ).

\section{The packing measure of the Sierpinski gasket}

\subsection{Code space and the overlapping set}

Iterating the basic identity (2) we obtain

$$
F^{k}(S)=\bigcup_{i \in M^{k}} f_{i}(S), k \in \mathbb{N}
$$

where $f_{i}:=f_{i_{1}} \circ f_{i_{2}} \circ \ldots \circ f_{i_{k}}$ for $i=i_{1} i_{2} \ldots i_{k} \in M^{k}$. Thus, when $k$ increases, the Sierpinski gasket decomposes into small, similar, copies of itself, $f_{i}(S), i \in M^{k}$, called cylinder sets of the $k$ th generation.

Given $A \subset \mathbb{R}^{2}$ and $i \in M^{k}$, we write $A_{i}=f_{i}(A)$, so the cylinder sets of the $k$ th generation are denoted by $S_{i}$. For $x \in S$ and $k \in \mathbb{N}$, there exists an $i_{\mid k}^{x}:=i_{1}^{x} i_{2}^{x} \ldots i_{k}^{x}$ such that $x \in S_{i_{\mid k}^{x}}, S_{i_{\mid k+1}^{x}} \subset S_{i_{\mid k}^{x}}$, and $x=\cap_{k=1}^{\infty} S_{i_{\mid k}}$. The mapping $\pi: M^{\infty} \rightarrow S$ defined by

$$
\pi(i)=\cap_{k=1}^{\infty} S_{i_{\mid k}}, i \in M^{\infty},
$$

gives a natural codification, through the codes in $M^{\infty}$, of the points in $S$. The codes are unique except for a countable subset $O \subset S$ called the overlapping set. We shall briefly examine this set, as it plays a role in our numerical procedure. Observe that $x \in S_{i} \cap S_{j} \neq \varnothing$ for $i, j \in M$ with $i \neq j$ implies $x \in O^{(1)}$ (see figure 2(a)), which is the set of the midpoints of the sides of the equilateral triangle $T$. This is the primary overlapping set, namely, the set of points each with two codes in $M^{\infty}$ differing in their first entries. In particular, if we adopt the convention $\widehat{i}=i i i i i \cdots \in M^{\infty}$ (so the fixed point $z_{i}$ of $f_{i}$ satisfies $\left.\pi(\widehat{i})=z_{i}, i \in M\right)$, then $\pi(0 \widehat{1})=\pi(\widehat{10}), \pi(0 \widehat{2})=\pi(2 \widehat{0})$, and $\pi(\widehat{1})=\pi(2 \widehat{1})$. Analogously, the $n$ ary overlapping set, $O^{(n)}$, that is, the set of points with two codes differing in their $n$th entries, can be written as

$$
O^{(n)}=F^{n-1}\left(O^{(1)}\right)=\left\{\pi(i * 0 \widehat{1}), \pi(i * 0 \widehat{2}), \pi(i * \widehat{12}): i \in M^{n-1}\right\},
$$

where the symbol $*$ denotes concatenation of sequences, i.e., $i * 0 \widehat{1}=i_{1} i_{2} \ldots i_{n-1} 011111 \ldots$

Remark 3 Let $k \in \mathbb{N}, i=i_{1} i_{2} \ldots i_{k} \in M^{k}$, and $r_{i}:=\Pi_{j=1}^{k} r_{i_{j}}$ where $r_{i_{j}}$ is the contraction ratio of the similitude $f_{i_{j}}, i_{j} \in M$. By (4), the identity

$$
\mu\left(f_{i}(S)\right)=\mu\left(S_{i}\right)=\mathbf{M}^{k}\left(\mu\left(S_{i}\right)\right)=\frac{1}{3^{k}}=\frac{1}{2^{s k}}=r_{i}^{s}=r_{i}^{s} \mu(S)
$$


shows that $\mu$ scales on cylinder sets as $P^{s}$ and $\mathcal{H}^{s}$ restricted to $S$ do, so these measures are all multiples. Consequently,

$$
\mu(A)=\frac{\mathcal{H}^{s}(A \cap S)}{\mathcal{H}^{s}(S)} .
$$

The boundary of the open ball $B(x, d)$ will be denoted by $\partial B(x, d)=\left\{y \in \mathbb{R}^{2}:|x-y|=d\right\}$, where $|x-y|$ is the Euclidean distance between $x$ and $y$.

\subsection{The packing measure of the Sierpinski gasket as a maximum}

The main result of this section characterizes the packing measure of the Sierpinski gasket through a reduction of the set of candidates for optimal balls given in (5). This simplification will be used in Section 3 to obtain bounds on the value of $P^{s}(S)$. The actual values of these bounds, given in Section 3, will be computed by the algorithm described in Section 4.1. Lemma 4 collects some basic facts needed in the proof of Theorem 6.

Lemma 4 The following statements are true.

i) If $B(x, d) \subset f_{i}(\mathcal{R})$ for some $i \in M$, then

$$
\frac{(2 d)^{s}}{\mu(B(x, d))}=\frac{(4 d)^{s}}{\mu\left(B\left(f_{i}^{-1}(x), 2 d\right)\right)} .
$$

ii) If $g: \mathbb{R}^{2} \rightarrow \mathbb{R}^{2}$ is an isometry such that $g(S)=S$, then

$$
\mu(B(x, d))=\mu(g(B(x, d))) .
$$

\section{Proof.}

i) If $B(x, d) \subset f_{i}(\mathcal{R})$ then, by the scaling property of the Hausdorff measure,

$$
\begin{aligned}
\mu(B(x, d)) & =\frac{\mathcal{H}^{s}(B(x, d) \cap S)}{\mathcal{H}^{s}(S)}=\frac{\mathcal{H}^{s}\left(B(x, d) \cap S_{i}\right)}{\mathcal{H}^{s}(S)}= \\
& =\frac{\left(\frac{1}{2}\right)^{s} \mathcal{H}^{s}\left(f_{i}^{-1}(B(x, d) \cap S)\right)}{\mathcal{H}^{s}(S)}=\left(\frac{1}{2}\right)^{s} \mu\left(B\left(f_{i}^{-1}(x), 2 d\right)\right),
\end{aligned}
$$

which implies (8).

ii) If $g: \mathbb{R}^{2} \rightarrow \mathbb{R}^{2}$ is an isometry such that $g(S)=S$, then

$$
\mu(g(B(x, d)))=\frac{\mathcal{H}^{s}(g(B(x, d)) \cap S)}{\mathcal{H}^{s}(S)}=\frac{\mathcal{H}^{s}(g(B(x, d)) \cap g(S))}{\mathcal{H}^{s}(S)}=\mu(B(x, d)) .
$$

Lemma 4 shows two basic senses in which the density of a given ball is invariant. This invariance (see Definition 5) together with the geometry of the Sierpinski gasket, will be used in the proof of Theorem 6 to remove sets of balls with repeated densities from (5).

Definition 5 Given $B(x, d) \subset \mathcal{R}$, we say that $B\left(x^{\prime}, d^{\prime}\right)$ is density equivalent to $B(x, d)$ if $B\left(x^{\prime}, d^{\prime}\right) \subset \mathcal{R}$ and

$$
\frac{(2 d)^{s}}{\mu(B(x, d))}=\frac{\left(2 d^{\prime}\right)^{s}}{\mu\left(B\left(x^{\prime}, d^{\prime}\right)\right)}
$$

Theorem 6 Let $S$ be the attractor of the system $\Psi=\left\{f_{0}, f_{1}, f_{2}\right\}$ given in (1). Then

$$
P^{s}(S)=\max \left\{\frac{(2 d)^{s}}{\mu(B(x, d))}: x \in S_{01}, d \geq \frac{\sqrt{3}}{16}, \quad \text { and } B(x, d) \subset \mathcal{R}\right\} .
$$


Proof. Our starting point is the characterization of the packing measure given in (5) with $\mathcal{O}=\mathcal{R}$, that is,

$$
P^{s}(S)=\sup \left\{\frac{(2 d)^{s}}{\mu(B(x, d))}: x \in S \quad \text { and } B(x, d) \subset \mathcal{R}\right\} .
$$

First, we show that (11) can be simplified to

$$
P^{s}(S)=\sup \left\{\frac{(2 d)^{s}}{\mu(B(x, d))}: x \in S_{0} \quad \text { and } B(x, d) \subset \mathcal{R}\right\} .
$$

This reduction is valid because any ball contained in $\mathcal{R}$ and centered in $S_{1} \cup S_{2}$ is density equivalent to another ball centered in $S_{0}$ (see Definition 5). This can be checked by noting that, on the one hand, any ball centeres in $S_{2}$ and contained $\mathcal{R}$ can be reflected across the altitude $h_{1}$ of the triangle $T$. On the other hand, any ball centered in $S_{1}$ and contained in $\mathcal{R}$ can be reflected across the altitude $h_{2}$ of the triangle $T$ (see figure 2 ). This yields, by (9), to density equivalent balls centered in $S_{0}$. This concludes the proof of (12) as it shows that any ball centered in $S_{1} \cup S_{2}$ and contained in $\mathcal{R}$ is density equivalent to another ball centered in $S_{0}$.

Next we show that (12) can be further reduced to

$$
P^{s}(S)=\sup \left\{\frac{(2 d)^{s}}{\mu(B(x, d))}: x \in S_{01} \quad \text { and } B(x, d) \subset \mathcal{R}\right\} .
$$

Let $B(x, d) \subset \mathcal{R}$ with $x \in S_{0}$. We divide the proof into three cases: $x \in S_{0 i}, i=0,1,2$. If $x \in S_{00}$, then $B(x, d) \subset f_{0}(\mathcal{R})$ and, hence, $(8)$ implies that $B\left(f_{0}^{-1}(x), 2 d\right)$ is density equivalent to $B(x, d)$. Observe that the previous argument can be repeated until $f_{0}^{-1}(x) \in S_{01} \cup S_{02}$. Now, if $x \in S_{02}$, then $h_{0}\left(S_{02}\right)=S_{01}$, where $h_{0}$ is the reflection across the altitude of $T$ through $z_{0}$. Thus, by $(9), B(x, d)$ is density equivalent to $B\left(h_{0}(x), d\right)$ (see figure 2(a)). This concludes the proof of (13).

Finally, we show that any ball with radius smaller than $\sqrt{3} / 16$ can be removed from the set of balls given in (13). To this end, let $B(x, d) \subset \mathcal{R}$ with $d<\sqrt{3} / 16$ and let $x \in S_{01}$. It suffices to show the existence of a ball density equivalent to $B(x, d)$ with center in $S_{01}$ and radius equal to $2 d$.

Consider the following three cases: $x \in S_{01 i}, i=0,1,2$.

If $x \in S_{010}$, then $B(x, d) \subset f_{0}(\mathcal{R})$ and therefore $B(x, d) \subset \mathcal{R}$ is density equivalent to $B\left(h_{2} \circ f_{0}^{-1}(x), 2 d\right)$ with $h_{2} \circ f_{0}^{-1}(x) \in S_{01}$, where $h_{2}$ is the reflection across the altitude $h_{2}$ of $T$ (see figure 2(a)).

If $x \in S_{012}$, then $\left.B(\tilde{\tau}(x), d)\right) \subset f_{2}(\mathcal{R})$ where $\tilde{\tau}: \mathbb{R}^{2} \rightarrow \mathbb{R}^{2}$ is the rotation of 240 degrees around the orthocenter of $T$ with $\tilde{\tau}(x) \in S_{201}$ (see figure 3 ). Therefore, $B\left(f_{2}^{-1} \circ \tilde{\tau}(x), 2 d\right)$ ) is density equivalent to $B(x, d)$ with $f_{2}^{-1} \circ \tilde{\tau}(x) \in S_{01}$.
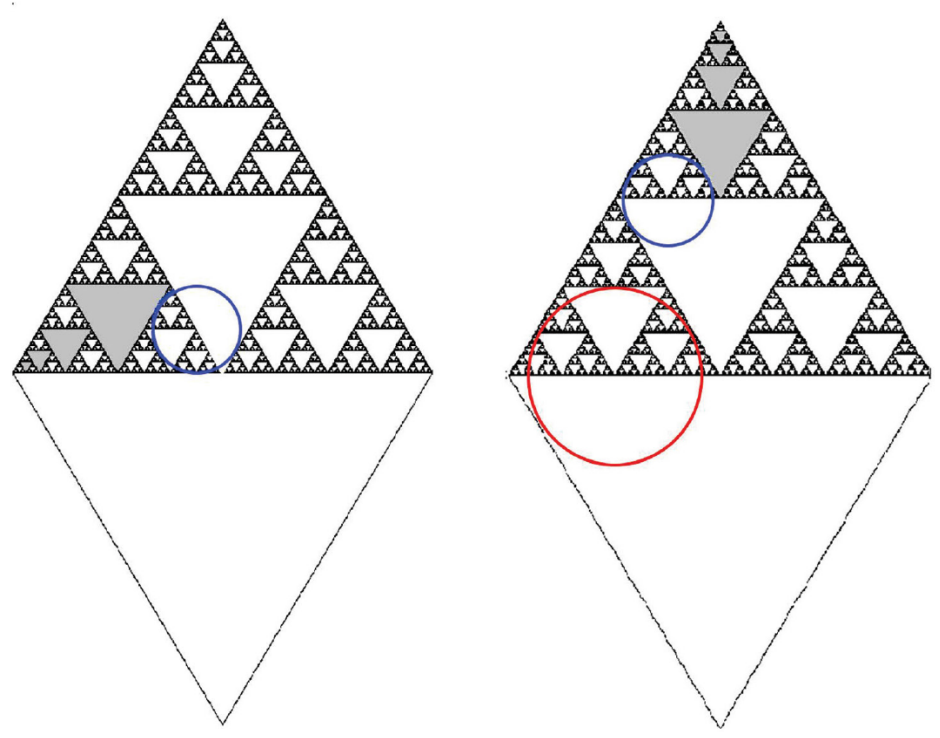

Figure 3. Rotation of 240 degrees around the orthocenter of $T$.

Now, if $x \in S_{011}$, let $L(x)=2 x-\left(\frac{1}{2}, 0\right)$ be the similarity with center $z=S_{0} \cap S_{1}$ and scale factor 2 . Then, as $d<\sqrt{3} / 16, B(x, d) \cap S=B(x, d) \cap\left(S_{01} \cup S_{10}\right)$. Moreover, $L\left(S_{01}\right)=S_{0}, L\left(S_{10}\right)=S_{1}$, and $B(L(x), 2 d) \cap S_{2}=\emptyset$ 
(see figure 4). Therefore, by (7) and the scaling property of the Hausdorff measure, we have that

$$
\begin{aligned}
\frac{(2 d)^{s}}{\mu(B(x, d))} & =\frac{\mathcal{H}^{s}(S)(2 d)^{s}}{\mathcal{H}^{s}(B(x, d) \cap S)}=\frac{\mathcal{H}^{s}(S)(2 d)^{s}}{\left(\frac{1}{2}\right)^{s} \mathcal{H}^{s}\left(L\left(B(x, d) \cap\left(S_{01} \cup S_{10}\right)\right)\right)}= \\
& =\frac{\mathcal{H}^{s}(S)(4 d)^{s}}{\mathcal{H}^{s}\left(B(L(x), 2 d) \cap\left(S_{0} \cup S_{1}\right)\right)}=\frac{(4 d)^{s}}{\mu(B(L(x), 2 d))} .
\end{aligned}
$$

This concludes the proof of the theorem as it proves that $B(x, d)$ is density equivalent to $B(L(x), 2 d)$ with $L(x) \in S_{01}$.

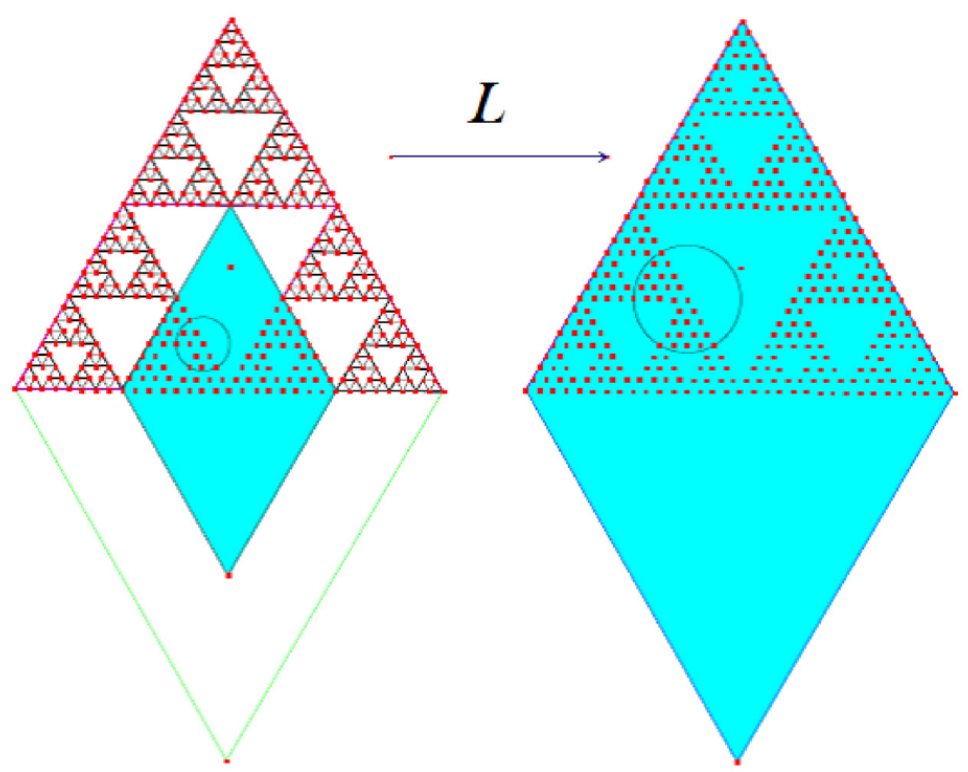

Figure 4. The similarity $L$ with center $z=S_{0} \cap S_{1}$ and scale factor 2 .

\section{Bounds for the packing measure}

In this section we show that a suitable discrete version of (10) can be used to estimate the value of $P^{s}(S)$. The aim is to construct two sequences, $\left\{P_{k}^{\text {sup }}\right\}_{k=1}^{\infty}$ and $\left\{P_{k}^{\inf }\right\}_{k=1}^{\infty}$, of discrete densities (see (15) and (16)), bounding the value of the density maximizing (10).

The discretization is made using a sequence $\left\{A_{k}\right\}_{k=1}^{\infty}$ of points such that $\overline{\cup_{k=1}^{\infty} A_{k}}=S$, where $\bar{A}$ is the closure of $A$, and a sequence $\left\{\mu_{k}\right\}_{k=1}^{\infty}$ of discrete probability measures, such that $\mu_{k}$ is supported on $A_{k}$, converging weakly to $\mu$.

The sequences $\left\{A_{k}\right\}$ and $\left\{\mu_{k}\right\}$ are defined as follows. Let $A_{1}=\left\{z_{0}, z_{1}, z_{2}\right\}$, where $z_{i}=f_{i}\left(z_{i}\right)$ is the fixed point of the similitude $f_{i}, i=0,1,2$. For $k>1, A_{k}:=F^{k-1}\left(A_{1}\right)$ and $\mu_{k}$ is the measure supported on $A_{k}$ and defined by

$$
\begin{aligned}
\mu_{k}:=\quad \mathbf{M}^{k-1}\left(\mu_{1}\right)=\frac{1}{3^{k-1}} \sum_{i \in M^{k}} \mu_{1} \circ f_{i}^{-1} \\
=\frac{1}{3^{k}} \sum_{i \in M^{k}} \frac{1}{3}\left(\delta_{f_{i}\left(z_{0}\right)}+\delta_{f_{i}\left(z_{1}\right)}+\delta_{f_{i}\left(z_{2}\right)}\right),
\end{aligned}
$$

where $\mu_{1}:=\frac{1}{3}\left(\delta_{z_{0}}+\delta_{z_{1}}+\delta_{z_{2}}\right)$ and $\delta_{x}$ is the unit mass at $x$. 
Note that $A_{2}=A_{1} \cup O^{(1)}$ and

$$
\begin{aligned}
A_{k} & =F^{k-2}\left(A_{2}\right)=F^{k-2}\left(A_{1} \cup O^{(1)}\right) \\
& =A_{k-1} \cup O^{(k-1)}=A_{1} \cup \bigcup_{j=1}^{k-1} O^{(j)}, \quad k=2,3, \ldots
\end{aligned}
$$

The discrete measures $\delta_{f_{i}\left(z_{j}\right)}, i \in M^{k}, j \in M$, occurring in (14) appear exactly twice at the overlap points in $O^{(p)}, p=1, \ldots, k-1$,

$$
f_{i}\left(z_{j}\right)=\pi\left(i_{1} i_{2} \ldots i_{p-1} i_{p} * \widehat{j}\right)=\pi\left(i_{1} i_{2} \ldots i_{p-1} j * \widehat{i_{p}}\right), i_{p} \neq j
$$

and only once for the points $z_{i}=f_{i_{1} i_{2} \ldots i_{k}}\left(z_{i}\right), i_{j}=i \in M, j=1,2, \ldots, k-1$, in $A_{1}$. Thus we can write

$$
\mu_{k}=\frac{1}{3^{k}}\left(\delta_{z_{0}}+\delta_{z_{1}}+\delta_{z_{2}}\right)+\frac{2}{3^{k}} \sum_{x \in A_{k}-A_{1}} \delta_{x}
$$

If $A_{1} \cap A=\varnothing$ then

$$
\mu_{k}(A)=\frac{2}{3^{k}} \sum_{x \in A_{k}} \delta_{x}(A)
$$

The sequences $\left\{P_{k}^{\text {inf }}\right\}_{k=1}^{\infty}$ and $\left\{P_{k}^{\text {sup }}\right\}_{k=1}^{\infty}$ are defined by

$$
\begin{aligned}
P_{k}^{\text {sup }}:=\quad & \max \left\{\frac{(2 r)^{s}}{\mu_{k}(B(x, r))}: \quad r=|x-y|, x \in A_{k} \cap S_{01},\right. \\
& \left.y \in A_{k} \backslash S_{2}, \quad \text { and } \quad \frac{\sqrt{3}}{16}-2^{2-k} \leq r<d(x, \partial \mathcal{R})\right\}
\end{aligned}
$$

and

$$
P_{k}^{\inf }:=\frac{\left(2 r_{k}\right)^{s}}{\mu_{k}\left(B\left(x_{k}, r_{k}+2^{-k}\right)\right)},
$$

where, for every $k \geq 1, B\left(x_{k}, r_{k}\right)$ denotes the ball maximizing $(15)$ and $d(x, \partial \mathcal{R}):=\inf \{|x-y|: y \in \partial \mathcal{R}\}$.

Theorem 7 For every $k \geq 6$,

$$
P_{k}^{\mathrm{inf}} \leq P^{s}(S) \leq K_{k} P_{k}^{\text {sup }}
$$

where

$$
K_{k}=\left(1-\frac{2^{6-k}}{\sqrt{3}}\right)^{-s}
$$

and the bounds $P_{k}^{\text {sup }}$ and $P_{k}^{\text {inf }}$ are given by (15) and (16), respectively.

Proof. Let $k \geq 6$, and let $B\left(x_{k}, r_{k}\right)$ be a ball of maximal density in the sense of (15).

The inequality $P_{k}^{\text {inf }} \leq P^{s}(S)$ is immediate because $B\left(x_{k}, r_{k}+2^{-k}\right)$ contains every cylinder set of generation $k$ intersecting $B\left(x_{k}, r_{k}\right)$ and hence

$$
\mu\left(B\left(x_{k}, r_{k}\right)\right) \leq \mu_{k}\left(B\left(x_{k}, r_{k}+2^{-k}\right)\right) .
$$

Moreover, as $B\left(x_{k}, r_{k}\right) \subset \mathcal{R},(5)$ and (19) imply

$$
P^{s}(S) \geq \frac{\left(2 r_{k}\right)^{s}}{\mu\left(B\left(x_{k}, r_{k}\right)\right)} \geq \frac{\left(2 r_{k}\right)^{s}}{\mu_{k}\left(B\left(x_{k}, r_{k}+2^{-k}\right)\right)} .
$$

We turn now to proving the inequality $P^{s}(S) \leq K_{k} P_{k}^{\text {sup }}$. Let $B(x, d) \subset \mathcal{R}$ be such that

$$
P^{s}(S)=\frac{(2 d)^{s}}{\mu(B(x, d))}
$$

with $x \in S_{01}, d \geq \sqrt{3} / 16$, and $B(x, d) \cap S_{2}=\emptyset$ (see Theorem 6).

We show first that, if there exist $y \in A_{k} \cap S_{01}$ and $z \in A_{k} \backslash S_{2}$ such that

$$
r:=|y-z| \in\left[d-2^{2-k}, d-2^{1-k}\right],
$$




$$
B(y, r) \subset B(x, d) \subset \mathcal{R},
$$

and

$$
\mu_{k}(B(y, r)) \leq \mu(B(x, d)),
$$

then $P^{s}(S) \leq K_{k} P_{k}^{\text {sup }}$. Note that $d-2^{2-k} \geq 0$ for every $d \geq \sqrt{3} / 16$ and $k \geq 6$.

Suppose that (21) and (22) hold. Then (15) implies that

$$
P_{k}^{\mathrm{sup}} \geq \frac{(2 r)^{s}}{\mu_{k}(B(y, r))}
$$

Now, (20), (23), (24), and (21) yield

$$
\begin{aligned}
P^{s}(S) & =\frac{(2 d)^{s}}{\mu(B(x, d))} \leq \frac{d^{s}(2 r)^{s}}{r^{s} \mu_{k}(B(y, r))} \leq\left(\frac{d}{r}\right)^{s} P_{k}^{\text {sup }} \leq\left(\frac{d}{d-2^{2-k}}\right)^{s} P_{k}^{\text {sup }} \\
& \leq\left(1-\frac{2^{2-k}}{d}\right)^{-s} \quad P_{k}^{\text {sup }} \leq K_{k} P_{k}^{\text {sup }},
\end{aligned}
$$

where $K_{k}$ is given by (18).

Next, we show the existence of $y \in A_{k} \cap S_{01}$ and $z \in A_{k} \backslash S_{2}$ satisfying (21), (22), and (23). We denote by $T_{k}$ the set of equilateral triangles having side length $2^{-k}$ and containing the cylinder sets of generation $k$. That is, for all $k \geq 1$,

$$
T_{k}:=F\left(T_{k-1}\right),
$$

with $T_{0}:=T$ being the equilateral triangle of side length one and vertices the three fixed points of the similarities in $\Psi$. Note that the three vertices of a triangle in $T_{k-1}$ belong to $A_{k}$. Now, $x$ belongs to a certain cylinder set of generation $k$ contained in $S_{01}$. Let $y$ be the unique vertex of such a cylinder set belonging to $A_{k}$. The distance of a point in a cylinder set to one of its vertices cannot exceed its diameter, hence

$$
|x-y| \leq 2^{-k} \text {. }
$$

Thus, for every $0<t \leq d-2^{1-k}$,

$$
B(y, t) \subset B\left(y, t+2^{-k}\right) \subset B(x, d) \subset \mathcal{R},
$$

whence,

$$
\mu_{k}(B(y, t)) \leq \mu\left(B\left(y, t+2^{-k}\right)\right) \leq \mu(B(x, d)),
$$

which proves (23) and (22), provided that (21) holds.

To conclude the proof there remains to show (21). In order to do so, let $L_{k}$ be the set formed by the edges of the triangles in $T_{k-1}$. Note that $L_{k}$ is a polygonal curve with vertices in $A_{k}$ and connected by edges of length $2^{-k+1}$. Let $z^{\prime} \in A_{k} \cap B\left(y, d-2^{-k+1}\right)^{c}$. That $A_{k} \cap B\left(y, d-2^{1-k}\right)^{c}$ is nonempty is true because it contains the set $A_{1}$ comprising the three vertices of $T_{0}$, since a ball $B(x, d) \subset \mathcal{R}$ cannot contain any point in $A_{1}$. Consider now the polygonal curve contained in $L_{k}$, beginning in $y$ and ending in $z^{\prime}$. Denote by $L$ the first side of such a polygonal curve that intersects $\partial B\left(y, d-2^{1-k}\right)$. Let $z \in A_{k}$ be the endpoint of $L$ contained in $B\left(y, d-2^{1-k}\right)$ and set

$$
r:=|y-z| \geq d-2^{1-k}-2^{1-k}=d-2^{2-k} .
$$

Observe that the existence of $z \neq y$ such that $z \in A_{k} \cap B\left(y, d-2^{1-k}\right)$ is guaranteed since $d-2^{2-k}>0$ for $k \geq 6$.

Finally, $z \in A_{k} \backslash S_{2}$ because $z \in B\left(y, d-2^{1-k}\right) \subset B(x, d)$ and $B(x, d) \cap S_{2}=\emptyset$. This concludes the proof of (21). 


\section{The algorithm}

In this section we describe an algorithm for computing the values $P_{k}^{\text {sup }}$ and $P_{k}^{\text {inf }}$ given in Theorem 7 and, hence, for obtaining numerical estimates of the packing measure of the Sierpinski gasket (see Section 4.3). The corresponding pseudocode is given in Section 4.2 .

\subsection{Description of the algorithm}

In general terms, the algorithm is based on searching for a ball, $B(x, d) \subset \mathcal{R}$, of maximal density $\frac{(2 d)^{s}}{\mu_{k}(B(x, d))}$, where $k=k_{\max }$ and $d=|x-y|$, with $k_{\max }$ being the largest integer permitted by the computational capacity available $\left(k_{\max }=15\right.$ in our case $)$ and $x, y \in A_{k}$. To this end the distances from each $x \in A_{k} \cap S_{01}$ to each point $y$ in $A_{k} \backslash S_{2}$ are computed. These distances will serve to compute the term $(2 d)^{s}$ in the density of $B(x, d)$ and also to compute $\mu_{k}(B(x, d))$. By $(14)$, this last computation essentially amounts to counting the points of $A_{k} \backslash S_{2}$ in $B(x,|x-y|)$. If the distances $|x-y|, y \in A_{k} \backslash S_{2}$, are sorted in increasing order, then the position of $|x-y|$ in the resulting list essentially gives the value of $\mu_{k}(B(x,|x-y|))$; we give the details below. We then take the ball of maximum density at $x$ and let $x$ vary over $A_{k} \cap S_{01}$, thereby finding the value of $P_{k}^{\text {sup }}$. Once $P_{k}^{\text {sup }}$ is known, the selected ball giving the optimal density in (15) is used to determine the lower bound according to (16).

We now give some details of the computation of the measures $\mu_{k}(B(x, d))$. Let $x \in A_{k} \cap S_{01}$. The set of balls centered at $x$ needed to compute $P_{k}^{\text {sup }}$ (see (15)) can be indexed by the set $D(x)$ of endpoints $z \in A_{k} \backslash S_{2}$ of their radii,

$$
D(x):=\left\{z \in A_{k} \backslash S_{2}: d_{0} \leq|z-x| \leq d(x, \partial \mathcal{R})\right\}
$$

where $d_{0}:=\frac{\sqrt{3}}{16}-2^{2-k}$. Let

$$
\begin{aligned}
U_{x}:=\quad \#\{z: & \left.z \in A_{k} \backslash S_{2} \text { and }|z-x|<d_{0}\right\}, \\
n_{D(x)}:= & U_{x}+\# D(x),
\end{aligned}
$$

and consider an ordering $\left\{z_{j}\right\}_{U_{x}<j \leq n_{D(x)}}$ of the set $D(x)$ such that the sequence of distances

$$
\widetilde{D}(x):=\left\{\left|z_{j}-x\right|\right\}_{U_{x}<j \leq n_{D(x)}}
$$

be nondecreasing. Now, to compute $\mu_{k}\left(B\left(x, \widetilde{d}_{j}\right)\right)$ with $\widetilde{d}_{j}:=\left|z_{j}-x\right| \in \widetilde{D}(x)$, we have to take into account that $B\left(x, \widetilde{d}_{j}\right)$ is an open ball, and the points of $A_{k} \backslash S_{2}$ in its boundary must be removed. Then, by (14), for any $j, U_{x}<j \leq n_{D(x)}$,

$$
\mu_{k}\left(B\left(x, \widetilde{d}_{j}\right)\right)=\frac{2 t_{x}(j)}{3^{k}}
$$

where

$$
t_{x}(j)=\min \left\{l: \widetilde{d}_{l}=\widetilde{d}_{j}, \widetilde{d}_{l} \in \widetilde{D}(x)\right\}-1
$$

Having obtained

$$
P\left(k, x, \widetilde{d}_{j}\right):=\frac{\left(2 \widetilde{d}_{j}\right)^{s}}{\mu_{k}\left(B\left(x, \widetilde{d}_{j}\right)\right)},
$$

for each $\widetilde{d}_{j} \in \widetilde{D}(x)$, we calculate

$$
P(k, x):=\max \left\{P\left(k, x, \tilde{d}_{j}\right): \quad U_{x}<j \leq n_{D(x)}\right\} .
$$

Repeating the same procedure for any $x \in A_{k} \cap S_{01}$, we get

$$
P_{k}^{\text {sup }}:=\max _{x \in A_{k} \cap S_{01}} P(k, x),
$$

the upper bound, $K_{k} P_{k}^{\text {sup }}$, for $P^{s}(S)$ as well as the center and the radius of a ball giving the optimal density (denoted by $z^{*}, d^{*}$, and $B\left(z^{*}, d^{*}\right)$, respectively). Finally, $P_{k}^{\text {inf }}$ is calculated using that $\mu_{k}\left(B\left(z^{*}, d^{*}+2^{-k}\right)\right)=$ $\frac{2}{3^{k}}\left(\#\left(B\left(z^{*}, d^{*}+2^{-k}\right) \cap A_{k}\right)\right)$. 


\subsection{Pseudocode}

Next, we give pseudocode for the algorithm computing the upper and lower bounds of $P^{s}(S)$ (see (15) and (16)). All the calculations are made using double-precision arithmetic.

\section{Initialization.}

- Fix $k=k_{\max }$ and define $s:=\frac{\ln 3}{\ln 2}$ and $d_{0}:=\frac{\sqrt{3}}{16}-2^{2-k}$.

- Set $P_{k}=0$ and $K_{k}=\left(1-\frac{2^{6-k}}{\sqrt{3}}\right)^{-s}$.

2. Generation of $A_{k} \backslash S_{2}$ and storage of the indices of the points in $A_{k} \cap S_{01}$.

2.1 Generate the sequence $A_{k} \backslash S_{2}=\left\{z_{i}\right\}_{i=1}^{n}$ where $n:=\# A_{k} \backslash S_{2}$.

2.2 Define $n_{S_{01}}:=\#\left(A_{k} \cap S_{01}\right)$ and store the sequence of indices, $\left\{I_{S_{01}}(j)\right\}_{1 \leq j \leq n_{S_{01}}}$, of the points in $A_{k} \cap S_{01}$, that is

$$
I_{S_{01}}(p)=j \quad \text { if } z_{j} \text { is the } p t h \text { point of } A_{k} \text { belonging to } S_{01} .
$$

\section{Computation of $P_{k}^{\text {sup }}$.}

For each $i=1, \ldots, n_{S_{01}}$ :

3.1 Compute and order the distances corresponding to the $i$ th point of $A_{k} \cap S_{01}$ in the following way. Let

$$
i^{*}:=I_{S_{01}}(i)
$$

be index of the $i$ th point of $A_{k} \cap S_{01}$. Compute the distances $\left\{\left|z_{j}-z_{i^{*}}\right|, 1 \leq j \leq n\right\}$ and store both the value

$$
U_{z_{i^{*}}}:=\#\left\{\left|z_{j}-z_{i^{*}}\right|: \quad\left|z_{j}-z_{i^{*}}\right|<d_{0}, \quad 1 \leq j \leq n\right\}
$$

and the sequence of distances corresponding to the points in

$$
D\left(z_{i^{*}}\right):=\left\{z_{j}: \quad d_{0} \leq\left|z_{j}-z_{i^{*}}\right|<d\left(z_{i^{*}}, \partial \mathcal{R}\right), \quad 1 \leq j \leq n\right\} .
$$

Set $n_{D\left(z_{i^{*}}\right)}:=U_{z_{i^{*}}}+\# n_{D\left(z_{i}^{*}\right)}$ and consider the sequence $\left\{s_{j}\right\}_{{z_{i^{*}}}<j \leq n_{D\left(z_{i^{*}}\right)}}$ of points in $D\left(z_{i^{*}}\right)$ sorted so that the sequence of distances $\widetilde{D}\left(z_{i^{*}}\right):=\left\{\widetilde{d}_{j}:=\left|s_{j}-z_{i^{*}}\right|\right\}_{U_{z_{i^{*}}<j \leq n_{D\left(z^{*}\right)}}}$ is nondecreasing. Obtain the corresponding set of indices $\{I(j)\}_{U_{z_{i^{*}}}<j \leq n_{D\left(z_{i}\right)}}$ induced by the previous ordering, that is, $s_{j}=z_{I(j)}$.

3.2 Use the sequence $\widetilde{D}\left(z_{i^{*}}\right)$ to compute the following items:

- For each $\widetilde{d}_{j} \in \widetilde{D}\left(z_{i^{*}}\right), j=U_{z_{i^{*}}}+1, \ldots, n_{D\left(z_{i^{*}}\right)}$, define

$$
h:=\frac{\left(2 \widetilde{d}_{j}\right)^{s}}{\mu_{k}\left(B\left(z_{i^{*}}, \widetilde{d}_{j}\right)\right)},
$$

where

$$
\mu_{k}\left(B\left(z_{i^{*}}, \widetilde{d}_{j}\right)\right)=\frac{2 t_{z_{i^{*}}}(j)}{3^{k}}
$$

and

$$
t_{z_{i^{*}}}(j):=\min \left\{l: \widetilde{d}_{l} \in \widetilde{D}\left(z_{i^{*}}\right), \quad \widetilde{d}_{l}=\widetilde{d}_{j}\right\}-1 .
$$

If $h>P_{k}$, set

$$
P_{k}=h, \quad I_{C}=i^{*}, \text { and } \quad I_{R}=I(j) .
$$

Observe that (31) is used to store the indices $I_{C}$ and $I_{R}$ of the points that characterize the ball $B\left(z_{I_{C}}, d\right)$ with $d=\left|z_{I_{R}}-z_{I_{C}}\right|$ corresponding to the current $P_{k}$.

4. Bounds for $P^{s}(S)$.

- Set $P_{k}^{\text {sup }}=P_{k}$ and compute the upper bound $K_{k} P_{k}^{\text {sup }}$ for $P^{s}(S)$. 
- Once the ball $B\left(z^{*}, d^{*}\right)$, with $z^{*}=z_{I_{C}}$ and $d^{*}=\left|z_{I_{R}}-z_{I_{C}}\right|$ has been selected among those giving the value of $P_{k}^{\text {sup }}$, use it to compute the lower bound for $P^{s}(S)$,

$$
P_{k}^{\inf }=\frac{(2 d)^{s}}{\mu_{k}\left(B\left(z^{*}, d^{*}+2^{-k}\right)\right)},
$$

where $\mu_{k}\left(B\left(z^{*}, d^{*}+2^{-k}\right)\right)=\frac{2}{3^{k}}\left(\#\left(B\left(z^{*}, d^{*}+2^{-k}\right) \cap A_{k}\right)\right)$.

It is important to note that, although for simplicity on the description of the pseudocode, the required computations are done for a fixed iteration $k$, the design of the algorithm permits simultaneous computation of all the items needed on each iteration $k=3, \ldots, k_{\max }$. Moreover, a decrease in computing time and memory space is possible since most of the distances that have been stored and sorted at $k=k_{\max }$ can be used for any $k<k_{\max }$.

\subsection{Numerical results}

The computer codes have been written in Fortran 90 and run on the HPC of the Complutense University of Madrid (see www.campusmoncloa.es/es/infraestructuras/eolo for technical description).

Table 1 shows the algorithm's output from the sixth to the fifteenth iteration.

Table 1. Algorithm output for $k=1, \ldots, 15$ : values of the radius $d(k)$ of the selected balls, lower and upper bounds and the discrete density $P_{k}^{\text {sup }}$.

\begin{tabular}{lllll}
\hline & $d(k)$ & $P_{k}^{\text {inf }}$ & $P_{k}^{\text {sup }}$ & $K_{k} P_{k}^{\text {sup }}$ \\
\hline$k=6$ & 0.054126587737 & 0.977078545264 & 2.149572799581 & 8.417030225302 \\
$k=7$ & 0.078125000000 & 1.406921618757 & 1.747993526335 & 2.999207461255 \\
$k=8$ & 0.148437500000 & 1.590092991382 & 1.679361369846 & 2.150018581504 \\
$k=9$ & 0.160156250000 & 1.640957358654 & 1.674896497407 & 1.886029238338 \\
$k=10$ & 0.160928492618 & 1.651285146818 & 1.671580901439 & 1.771841897590 \\
$k=11$ & 0.160156250000 & 1.660396880844 & 1.670290388098 & 1.719192691100 \\
$k=12$ & 0.160441818577 & 1.664080807032 & 1.668639759055 & 1.692779349798 \\
$k=13$ & 0.160621711014 & 1.665943279480 & 1.668558608163 & 1.680557136923 \\
$k=14$ & 0.160543021274 & 1.667178755724 & 1.668305166018 & 1.674285995048 \\
$k=15$ & 0.160549599562 & 1.667728055374 & 1.668272620403 & 1.671258613367 \\
\hline
\end{tabular}

Theorem 7 together with the output of the algorithm for $k=15$ gives

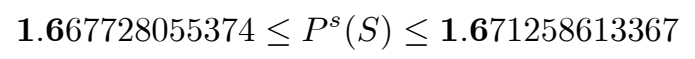

and a $100 \%$ confidence interval of length less than 0.003531. Moreover, in every iteration in the range $k=6, \ldots, 15$, the selected ball, $B\left(z_{I_{C}(k)}, d(k)\right)$, is centered in $z_{I_{C}(k)}=(0.5,0)$ and the radius $d(k)$ varies slightly from the ninth iteration on. In this sense we observe stability in the results which indicates that a good approximation to the optimal ball giving the maximum value in $(10)$ should be $B((0.5,0), 0.1605)$.

The pattern observed in the optimal balls found algorithmically as a function of $k$ raises the problem of whether it exists a finite $k$ at which the $\mu_{k}$ density of the selected ball equals to $P^{s}(S)$, in which case the exact value of $P^{s}(S)$ can be computed. We say that a self-similar set with this characteristic enjoys the finite time computability property (see examples in [28]). Certain conditions are required for the finite time computability property to hold. Firstly, the optimal ball $B^{*}$ giving $P^{s}(S)$ should be centered in $A_{k}$ for a reasonably large $k$ depending on the constrains imposed by the available computing capacity. Moreover, $A_{k} \cap \partial B^{*}$ should be nonempty. These two conditions imply that the optimal ball can be found in finite time, however they do not guarantee that the exact value of $P^{s}(S)$ can be computed in finite time. Observe that estimating the exact value of $\mu\left(B^{*}\right)$ might require an infinite process unless $B^{*}$ can be written as a finite union of $k$ th generation cylinder sets. In this case, $\mu\left(B^{*}\right)=\mu_{j}\left(B^{*}\right)$ and $P_{j}^{\text {sup }}=P^{s}(S)$ for $j \geq k$. The results showed in Table 1 indicate that $S$ does not enjoy this property. 
Regarding the bounds for $P^{s}(S)$, notice that the number of fixed decimal places in the column of Table 1 corresponding to $P_{k}^{\text {inf }}$ varies from two (in every iteration in the range $k=11, \ldots, 14$ ) to three (from the fourteenth iteration on). The stability behavior for $K_{k} P_{k}^{\text {sup }}$ is, however, worse than the one observed for the lower bound. This is mainly caused by the slow convergence to one of the term $K_{k}$ (see fig 5).

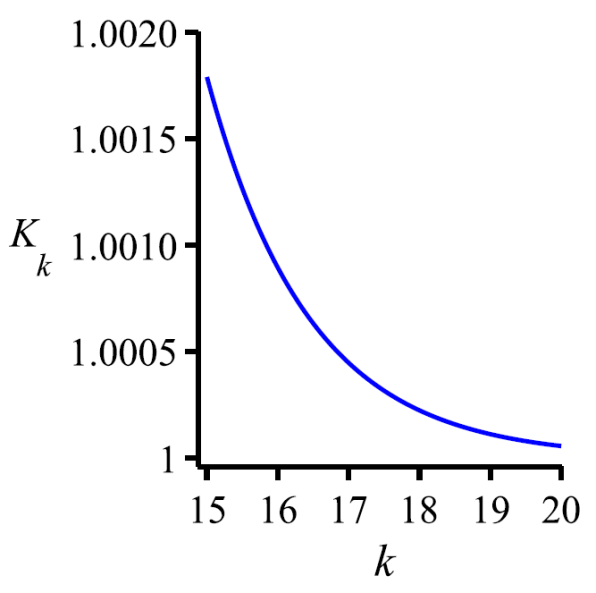

Figure 5. Convergence to one of the term $K_{k}=\left(1-\frac{2^{6-k}}{\sqrt{3}}\right)^{-s}$

Due to the fact that the values of $P_{k}^{\text {sup }}$ are stabilized around 1.668 from the twelfth iteration on, and since the lower and upper bounds are arbitrarily close to $P_{k}^{\text {sup }}$ for $k$ sufficiently large, we can conjecture that

$$
P^{s}(S) \sim 1.668 .
$$

More precise estimates for $P^{s}(S)$ would require either a significant increment of $k_{\max }$ (which at the moment is out of reach due to the memory and computational time requirements) or a refinement of the upper bound for $P^{s}(S)$.

Finally, a comparison of the current results (see (32) and (33)) with those obtained in [28] for the totally disconnected case leads to the open problem of the continuous dependence of the packing measure on the values of the contraction ratios. More precisely, let $S_{r}$ be the totally disconnected Sierpinski attractor with contraction ratio $r<0.5$ and let $P^{s(r)}\left(S_{r}\right)$ be the corresponding packing measure of $S_{r}$ with $s(r):=\frac{-\log 3}{\log (r)}$. Approximate values of $P^{s(r)}\left(S_{r}\right)$ as well as the corresponding upper and lower bounds can be found in [28] for a wide range of values of $r<0.5$. An interesting question is to know whether the estimates of $P^{s(r)}\left(S_{r}\right)$ given in [28] together with those of $P^{s}(S)$ obtained here support the conjecture of Qiu (see [34]) which implies that $P^{s(r)}\left(S_{r}\right)$ is continuous as a function of $r$ at $r=0.5$.

If $r<0.5$, a continuous behavior of $P^{s(r)}$ is supported by the estimate values obtained in [28] (see Figure 1 in [28]). This fact is in concordance with Qiu's result (see [34]) which establishes the continuity of the packing measure function of general self-similar sets satisfying the strong separation condition and, in particular, implies the continuity of $P^{s(r)}\left(S_{r}\right)$ for $r<0.5$. However, the estimate of $P^{s}(S)$ obtained in this note (see (33)) is about half the approximate value corresponding to $P^{s(r)}\left(S_{r}\right)$ for $r \sim 0.5$ (see [28]), which indicates that Qiu's conjecture might be false. The relation $P^{s}(S) \sim \frac{1}{2} P^{s(r)}\left(S_{r}\right)$ for $r \sim 0.5$ might be caused by the differences between the selected balls on each case. More precisely, the selected optimal ball for $P^{s(r)}\left(S_{r}\right)$ is density equivalent to another one centered in $(r, 0)$ and with radius less than $1-2 r$, that is, the distance separating the first generation cylinders sets. The case $r=0.5$ is different as such an optimal ball is centered at $(0.5,0)$, intersects two first generation cylinder sets and its measure is about twice the measure of the optimal ball corresponding to the disconnected case.

Acknowledgement 8 The computational part of this work was performed in EOLO, the HPC of Climate Change of the International Campus of Excellence of Moncloa, funded by the MECD and MICINN. This is a contribution to the CEI Moncloa.

We are grateful to the IMI-Institute of Interdisciplinary Mathematics, Universidad Complutense de Madrid, by the support received to our research. 


\section{References}

[1] Alexander, S. (1984) Some properties of the spectrum of the Sierpinski gasket in a magnetic field. Phys. Rev. B (3) 29, no. 10, 5504-5508.

[2] Baek, H. K. (2006) Packing dimension and measure of homogeneous Cantor sets. Bull. Austral. Math. Soc. 74 , no. 3, 443-448.

[3] Bárány, B.; Ferguson, A. and Simon, K. (2012) Slicing the Sierpinski gasket. Nonlinearity 25, 1753.

[4] Barlow, M. and Perkins, E. (1988) Brownian motion on the Sierpinski gasket. Probab. Theory Related Fields, 79, no. 4, 543-623.

[5] Bohl, T. and J. Zähle, M. (2013) Curvature-direction measures of self-similar sets. Geom. Dedicata 167, $215-231$.

[6] Dalrymple, K.; Strichartz, R. S. and Vinson, J. P. (1999) Fractal differential equations on the Sierpinski gasket. J. Fourier Anal. Appl. 5, no. 2-3, 203-284.

[7] Falconer, K. (1997) Techniques in Fractal Geometry. John Wiley.

[8] Falconer, K. and Hu, J. (1999) Non-linear elliptical equations on the Sierpinski gasket. J. Math. Anal. Appl. 240, no. 2, 552-573.

[9] Feng, D. (2003) Exact packing measure of linear Cantor sets, Math. Nachr. 248/249, 102-109.

[10] Feng, D.; Hua, J. G. and Wen, Z.J. (1999) Some relations between packing premeasure and packing measure. Bull. Lon. Math. Soc. 31, 6, 665-70.

[11] Fukushima, M. and Shima, T. (1992) On a spectral analysis for the Sierpinski gasket. Potential Anal. 1, no. $1,1-35$.

[12] Garcia, I. and Zuberman, L. (2012) Exact packing measure of central Cantor sets in the line. J. Math. Anal. Appl. 386, no. 2, 801-812.

[13] Gefen, Y.; Aharony, A.; Shapir, Y. and Mandelbrot, B. B. (1984) Phase transitions on fractals. II. Sierpinski gaskets. J. Phys. A 17, no. 2, 435-444.

[14] Hutchinson, J. E. (1984) Fractals and self-similarity. Ind. J. Math. 30, Ind. J. Math. 30, 713-747.

[15] Jia, B.; Luo J., Zhou, Z. and Zhu, Z. (2003) The packing measure of the Cartesian product of the middle third Cantor set with itself. J. Math. Anal. Appl., 288, no.2, 424-441.

[16] Jia, B. (2007) Bounds of Hausdorff measure of the Sierpinski gasket Journal of Mathematical Analysis and Applications, 330, 2, 1016-1024.

[17] Jia, B. and Zhiwei, Z. (2004) The packing measure of a class of generalized Sierpinski carpet. Analysis in Theory and Applications, 20, 1, 69-76.

[18] Jia, B.; Zhou, Z. and Zhu, Z. (2002) A lower bound for the Hausdorff measure of the Sierpinski gasket. Nonlinearity 15, no. 2, 393-404.

[19] Kigami, J. (2009) Volume doubling measures and heat kernel estimates on self-similar sets. Mem. Amer. Math. Soc., no.932.

[20] Kigami, J. (2001) Analysis on fractals. Cambridge Tracts in Mathematics, 143. Cambridge University Press, Cambridge.

[21] Lal, N. and Lapidus, M. L. (2012) Hyperfunctions and spectral zeta functions of Laplacians on selfsimilar fractals. J. Phys. A 45, no. 36, 365205, 14 pp.

[22] Lapidus, M. and Sarhad, J. (2014) Dirac operators and geodesic metric on the harmonic Sierpinski gasket and other fractal sets. J. Noncommut. Geom. 8 , no. 4, 947-985. 
[23] Le, A.; Gao, F.; Xi, L.; Yin, S. (2015) Complex networks modeled on the Sierpinski gasket. Phys. A 436, 646-657.

[24] Llorente, M. and Morán, M. (2007) Self-similar sets with optimal coverings and packings. J. Math. Anal. Appl. 334, 2, 1088-1095.

[25] Llorente, M. and Morán, M. (2010) Advantages of the centered Hausdorff measure from the computability point of view. Math. Scand. 107, no. 1, 103-122.

[26] Llorente, M. and Morán, M. (2012) An algorithm for computing the centered Hausdorff measures of self-similar sets. Chaos Solitons Fractals, 45 , no. 3, 246-255.

[27] Llorente, M. and Morán, M. (2016) Computability of the packing measure of totally disconnected selfsimilar sets. Ergodic Theory and Dynamical Systems, 36, 5, 1534-1556.

[28] Llorente, M.; Mera, M. and Morán, M. (2016) Rate of convergence: the packing and centered Hausdoff measures of totally disconnected self-similar sets. arXiv:1602.03567 [math.DS]

[29] Mattila, P. (1982) On the structure of self-similar fractals. Ann. Acad. Sci. Fenn. Ser. A I Math. 7, no. 2, 189-195.

[30] Mattila, P. (1995) Geometry of Sets and Measures in Euclidean Spaces. Cambridge University Press.

[31] Meinershagen, S. (2001/02) The Hausdorff meas $\div$ ure and the packing measure on a perturbed Cantor set. Real Anal. Exchange 27, no. 1, 177-190.

[32] Móra, P. (2013) Random and deterministic fractals. PhD Thesis. Budapest University of Technology and Economics.

[33] Morán M. (2005) Computability of the Hausdorff and packing measures on self-similar sets and the self-similar tiling principle. Nonlinearity 18, no. 2, 559-570.

[34] Qiu, H. (2012) Continuity of packing measure functions of self-similar iterated function systems, Ergodic Theory and Dynamical Systems 32, 3, 1101-1115.

[35] Strichartz, R. and Wong, C. (2004) The p-Laplacian on the Sierpinski gasket. Nonlinearity 17, 595.

[36] Sullivan, D. (1984) Entropy, Hausdorff measures old and new, and limit sets of geometrically finite Kleinian groups. Acta Math. 153 , no. 1, 259-277.

[37] Taylor, S. J. and Tricot, C. (1985) The packing measure and its evaluation for a Brownian path. Trans. Amer. Math. Soc. 288, no. 2, 679-699.

[38] Taylor, S. J. and Tricot, C. (1986) Packing measure of rectifiable subsets of the plane. Math. Proc.Cambridge Philos. Soc. 99, no. 2, 285-296.

[39] Teplyaev, A. (1998) Spectral analysis on infinite Sierpinski gaskets. J. Funct. Anal. 159, no. 2, 537-567.

[40] Tricot, C. (1980) Sur la classification des ensembles boréliens de mesure de Lebesgue nulle. These de doctorat, Genéve: Impremiere Nationale, 1921.

[41] Tricot, C. (1982) Two definitions of fractional dimension. Math. Proc. Cambridge Philos. Soc. 91, no. 1, $57-74$.

[42] Tricot, C. (2008) Geometries et mesures fractales: Une introduction. France, Ellipses.

[43] Zhou, Z. and Feng, L. (2000) A new estimate of the Hausdorff measure of the Sierpinski gasket. Nonlinearity 13, no. 3, 479-91.

[44] Zhou, Z. and Zhu, Z. (2007) The centered covering measures of a class of self-similar sets on the plane. Real Anal. Exchange 33, no. 1, 217-234.

[45] Zhu, Z. and Zhou, Z. (2002) The Hausdorff centred measure of the symmetry Cantor sets. Approx. Theory Appl. 18, no. 2, 49-57. 